Bentham open
CrossMark

\title{
Synthesis, Characterization and Antimicrobial Evaluation of Novel Mannich Bases Containing Pyrazole-5-One Phosphonates
}

\author{
V. E. Rani ${ }^{*}$ and L. K. Ravindranath \\ Department of Chemistry, S. K. University, Ananthapuramu, A.P, India
}

Received: April 3, 2015

Revised: April 5, 2016

Accepted: April 05, 2016

\begin{abstract}
:
Background:

Newly synthesised compounds of phosphonates were prepared by condensation of diethylphosphate with imine which undergoes a reaction of mannich bases with pyrazole containing schiffs base. The base was prepared by condensation of aldehyde with primary amine. These newly synthesised derivatives were characterised by spectral analysis.
\end{abstract}

\section{Objective:}

Mannich bases are very important to synthesize wide variety of natural products and pharmaceuticals.

\section{Method:}

Thin Layer Chromatography was performed on aluminum sheet of silica gel 60F254, E-Merk, Germany using iodine as visualizing agent. IR Spectra were recorded as KBr pellets on Perkin-Elmer 1000 units, instruments. All ${ }^{1} \mathrm{H}$ and ${ }^{13} \mathrm{C}$-NMR spectra were recorded on a Varian XL-300 spectrometer operating at $400 \mathrm{MHz}$ and $75 \mathrm{MHz} .{ }^{31} \mathrm{P}-\mathrm{NMR}$ spectra were recorded on a Varian XL-spectrometer operating at $161.89 \mathrm{MHz}$. The compounds were dissolved in dimethylsulfoxide and Chemical shifts were referenced to Trimethylsilane $\left({ }^{1} \mathrm{H}\right.$ and $\left.{ }^{13} \mathrm{C}-\mathrm{NMR}\right)$ and $85 \%$ phosphoric acid $\left({ }^{31} \mathrm{P}-\mathrm{NMR}\right)$.

Results:

Some of the novel synthetic compounds of Pyrazole Mannich base-Phosphonates showed great potential in field of medicinal chemistry and good biological activity.

\section{Conclusion:}

It can be concluded that this class of compounds certainly holds great potential for the discovery of novel classes of antimicrobial agents.

Keywords: Antibacterial, Antifungal, Phosphonates, Pyrazole-5-one.

\section{INTRODUCTION}

Pyrazole-5-one derivatives possess various types of biological activities. It is due to their wide use in medicinal chemistry some of them possess anti-tuberculosis, anti-neoplastic, anti-diabetic, anti-fertility, anti-thyroid and anti microbial activity [1 - 4].

The chemistry of phosphorus heterocyclic compounds containing nitrogen has pioneered the application of combinatorial techniques to the development of new pharmaceutical materials with novel properties. Organophosphorus compounds possess significant biological activity against broad spectrum of bacteria, pets, virus, fungicides and plant

\footnotetext{
* Address correspondence to this author at the Department of Chemistry, S. K. University, Ananthapuramu, A.P, India; E-mail: vesther9@gmail.com
} 
growth regulators. Some Organophosphorus compounds have been described in the literature as inhibitors of bacterial [5], herbicides, insecticides, pesticides [6, 7], anti-fungal agents [8], anti-HIV [9], anti-cancer[10], anti-viral and antiinflammatory[11].

A good deal of importance was given to Phosphonate derivatives in the field of organophosphorus heterocyclic chemistry due to their unique biological applications [12]. In view of the above observations, we synthesized Mannich bases containing Pyrazole-5-one Phosphonates and screening for possible biological and pharmacological activities.

\section{RESULTS AND DISCUSSION}

\section{Chemistry}

All the chemicals used in the present investigation were purchased from Sigma-Aldrich Chemicals company, Inc. USA. And used without further purification. TLC was performed on aluminium sheet of silica gel 60F254, E-Merk, Germany using iodine as visualizing agent. Melting points were determined in open capillary tubes on Mel-Temp apparatus and are uncorrected. Column chromatography was performed on silica gel with different solvent systems as eluents to afford the pure compound. The IR Spectra were recorded as KBr pellets on Perkin-Elmer 1000 units, instruments. All ${ }^{1} \mathrm{H}$ and ${ }^{13} \mathrm{C}-\mathrm{NMR}$ spectra were recorded on a Varian XL-300 spectrometer operating at $400 \mathrm{MHz}$ for ${ }^{1} \mathrm{H}$ -NMR and $75 \mathrm{MHz}$ for ${ }^{13} \mathrm{C}-\mathrm{NMR} .{ }^{31} \mathrm{P}-\mathrm{NMR}$ spectra were recorded on a Varian XL-spectrometer operating at 161.89MHz. The compounds were dissolved in DMSO- $\mathrm{d}_{6}$ and Chemical shifts were referenced to TMS $\left({ }^{1} \mathrm{H}\right.$ and ${ }^{13} \mathrm{C}$ $\mathrm{NMR}$ ) and $85 \% \mathrm{H}_{3} \mathrm{PO}_{4}\left({ }^{31} \mathrm{P}-\mathrm{NMR}\right)$. Mass spectral data was recorded on FAB-MS instrument at 70 ev with direct inlet system. Elemental analysis was recorded on a Carlo Erba 1108 elemental Analyzer, Central Drug Research Institute, Lucknow, India.

\section{CONCLUSION}

The newly synthesized compounds Diethyl ((4-fluorophenyl)/(4-chlorophenyl)/(4-bromophenyl)/(4-(trifluoromethyl) phenyl) amino) (3-methyl-1-(morpholino methyl)/(4-methylpiperazin-1-yl) -5- oxo-4, 5-dihydro-1H-pyrazol-4yl) methyl) phosphonates $\mathbf{6}(\mathbf{a}-\mathbf{h})$ were found to be active in the study of antifungal activity. It can be concluded that this class of compounds certainly holds great promise towards the pursuit to discover novel classes of antimicrobial agents.

\section{EXPERIMENTAL SECTION}

\section{General Procedure A for Preparation of 3(a-d)}

The quantity of 4-fluoro aniline (2.2gr, 0.020 mole) (2a) and 3 - Methyl - 5 - oxo - 4, 5 - dihydro - $1 \mathrm{H}$ - pyrazole - 4 - carbaldehyde (1.746gr, 0.014mole) (1) were dissolved in absolute alcohol, to this three drops of acetic acid is added then heated on a steam bath for 5-6 hours at $100^{\circ} \mathrm{C}$. After standing for 24 hours at room temperature, the crude product was purified by column chromatography (60-120 mesh silica gel,eluent: 10\% EtoAc pet ether). Finally, the product compound 4 - (((4 - fluorophenyl) imino) methyl) - 3 - methyl - 1H - pyrazol - 5(4H) - one (3a) which was recrystallized from warm absolute alcohol. Yield $75 \%, \mathrm{~m} \mathrm{p} 153-155^{\circ} \mathrm{C}$.

The similar procedure was adopted to synthesize 3(b-d) $(2.7 \mathrm{gr}, 0.011 \mathrm{~mol}-3 \mathrm{~b}, 3.1 \mathrm{gr}, 0.011 \mathrm{~mol}-3 \mathrm{c}, 2.9 \mathrm{gr}$, $0.018 \mathrm{~mol}-3 \mathrm{~d}$ ) by condensing 3-Methyl-5-oxo-4, 5-dihydro-1H-pyrazole-4-carbaldehyde (1) with 4-chloro aniline (2.5gr, $0.020 \mathrm{~mol}-2 \mathrm{~b})$, 4-bromo aniline (3.44gr, 0.20mol-2c) and 4-trifluoro aniline (3.22gr, 0.020mol-2d) respectively.

\section{General Procedure B for Preparation of 5(a-h)}

A mixture of 4-(((4-fluorophenyl) imino) methyl)-3-methyl-1H-pyrazol-5(4H)-one(0.87gr, 0.004mol) (3a), morpholine $(0.78 \mathrm{gr}, 0.09 \mathrm{~mol}) \mathbf{( 4 a )}(0.15 \mathrm{~mol})$ and water $20 \mathrm{ml}$ was stirred to obtained a clear solution. To this solution, HCHO (0.05 mol) and DMF were added in ice cold condition and stirred for 2 hours in an ice bath and left over night at room temperature. The progress of the reaction was monitored by TLC using cyclohexane and ethylacetate (7:3) solvent mixture as a mobile phase. At the end of the reaction, the mixture was taken in a $30 \mathrm{ml}$ dichloromethane and neutralized with $50 \mathrm{ml} 1 \mathrm{~N} \mathrm{NaOH}$ solution, after neutralization the mixture was extracted with $\mathrm{CH}_{2} \mathrm{Cl}_{2}(3(25 \mathrm{ml})$. The combined extract was dried on $\mathrm{Na}_{2} \mathrm{SO}_{4}$. After filtration, the solvent was removed with rota evaporator. The residue was purified by column chromatography, using 60-120 mesh silica and $\mathrm{CHCl}_{3}$ solvent was used as an elutent. Finally the product compound 4 - (((4 - fluorophenyl) imino) methyl) - 3 - methyl - 1 - (morpholinomethyl) - 1H - pyrazol - 5(4H) one(0.891gr, $0.0028 \mathrm{~mol})$ (5a) was purified from aqueous dimethyl formamide. Yield $70 \%$, $\mathrm{m} \mathrm{p} 162-164^{\circ} \mathrm{C}$. 
The similar procedure was adopted to synthesize $\mathbf{5}(\mathbf{b}-\mathbf{h}) \quad(1.305 \mathrm{gr}, 0.003 \mathrm{~mol}-\mathbf{5 b}, 1.32 \mathrm{gr}, 0.0035 \mathrm{~mol}-\mathbf{5 c}$, $1.28 \mathrm{gr}, 0.0035 \mathrm{~mol}-5 \mathrm{~d}, 1,15 \mathrm{gr}, 0.0035 \mathrm{~mol}-5 \mathrm{e}, 1.13 \mathrm{gr}, 0.0032 \mathrm{~mol}-5 \mathrm{f}, 1.27 \mathrm{gr}, 0.0032 \mathrm{~mol}-5 \mathrm{~g}, 1.23 \mathrm{gr}, 0.0032 \mathrm{~mol}-5 \mathrm{~h})$ by condensing 3(a-d) $(0.87 \mathrm{gr}, 0.004 \mathrm{~mol}-3 \mathrm{a}, 1.414 \mathrm{gr}, 0.006 \mathrm{~mol}-3 \mathbf{b}, 1.40 \mathrm{gr}, 0.005 \mathrm{~mol}-3 \mathbf{c}, 1.34 \mathrm{gr}, 0.005 \mathrm{~mol}-3 \mathrm{~d})$ with morpholine (0.78gr, $0.009 \mathrm{~mol})$ (4a) N-methylpiperazine (0.90gr, $0.005 \mathrm{~mol})$ (4b) respectively.

\section{General Procedure C for Preparation of 6(a-h) (Scheme-1)}

A mixture of 4-(((4-fluorophenyl) imino) methyl)-3-methyl-1-(morpholinomethyl)-1H- pyrazol-5(4H)-one $(0.95 \mathrm{gr}$, $0.003 \mathrm{~mol})(\mathbf{5 a})$ and Diethyl phosphite $(1.24 \mathrm{ml}, 0.009 \mathrm{~mol})$ in anhydrous toluene $(15 \mathrm{ml})$ was added dropwise. Stirring was continued at room temperature for another 0.5 hour, after which the mixture was heated under reflux for 4-6 hours. The reaction was monitored by TLC on silica gel using petroleum ether-ethyl acetate $(1: 2 \mathrm{v} / \mathrm{v})$. After completion of the reaction, the solvent was removed by rota evaporator and the resulting residue was purified by column chromatography on silicagel (100-200 mesh) and ethyl acetae-hexane, (3:7 ratio) as an eluent to afford pure Diethyl (((4-fluorophenyl) amino) (3-methyl -1- (morpholinomethyl) -5-oxo -4,5-dihydro-1H-pyrazol-4-yl) methyl) phosphonates(0.95gr, $0.002 \mathrm{~mol})$ (6a), was purified from aqueous dimethyl formamide. Yield $70 \%, \mathrm{~m} \mathrm{p} 176-178^{\circ} \mathrm{C}$.

The similar procedure was adopted to synthesize 6(b-h) $(0.96 \mathrm{gr}, 0.002 \mathrm{~mol}-\mathbf{6 b}, 1.03 \mathrm{gr}, 0.002 \mathrm{~mol}-6 \mathbf{c}, 0.98 \mathrm{gr}$, $0.001 \mathrm{~mol}-6 \mathrm{~d}, 0.98 \mathrm{gr}, 0.002 \mathrm{~mol}-6 \mathrm{e}, 1.00 \mathrm{gr}, 0.002 \mathrm{~mol}-\mathbf{6 f}, 1.00 \mathrm{gr}, 0.001 \mathrm{~mol}-6 \mathrm{~g}, 1.16 \mathrm{gr}, 0.002 \mathrm{~mol}-6 \mathrm{~h})$ by the reaction between 5(b-h) with Diethyl phosphite. The structure of these newly synthesized compounds of $\mathbf{6}(\mathbf{a}-\mathbf{h})$ were established by IR, ${ }^{1} \mathrm{H}-\mathrm{NMR},{ }^{13} \mathrm{C}-\mathrm{NMR},{ }^{31} \mathrm{P}-\mathrm{NMR}$, and mass data and elemental analysis.

Diethyl (((4-fluorophenyl) amino) (3 - methyl - 1 - (morpholinomethyl) - 5 - oxo - 4, 5 - dihydro-1H-pyrazol-4-yl) methyl) phosphonates $6(a)$ according to general procedure $\mathrm{C}$ to afford the target compound as a yellow solid (0.95gr)

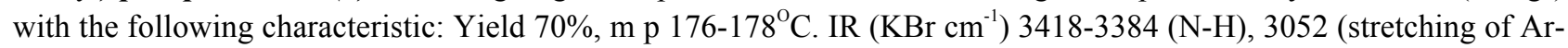
$\mathrm{H}), 2940$ and $2895\left(\mathrm{CH}_{3} \& \mathrm{CH}_{2}\right.$ of aliphatic- $\left.\mathrm{CH}\right), 1670(\mathrm{C}=\mathrm{O}), 1478-1375$ (stretching vibrations of pyrazolone ring), 1140 (C-O), $1245(\mathrm{P}=\mathrm{O}), 1100(\mathrm{C}-\mathrm{F})$ and $745 \mathrm{~cm}^{-1}$ (P-O). ${ }^{1} \mathrm{H}-\mathrm{NMR}$ (400MHz, DMSO-d6): ( $\mathrm{CH}_{2}-\mathrm{CH}_{3} \mathrm{~J}=7 \mathrm{~Hz}$ ), 1.94 (s, 3H, of $\mathrm{CH}_{3}$ group), 2.40 (d, $1 \mathrm{H}, \mathrm{CH}$ of pyrazolone ring), 2.50 (t, $4 \mathrm{H},-\mathrm{CH}_{2}-\mathrm{N}-\mathrm{CH}_{2}-\mathrm{of}$ morpholine ring $\mathrm{J}=7 \mathrm{~Hz}), 3.65\left(\mathrm{t}, 4 \mathrm{H},-\mathrm{CH}_{2}-\mathrm{O}-\mathrm{CH}_{2}\right.$-of morpholine ring $\left.\mathrm{J}=7 \mathrm{~Hz}\right), 4.10(\mathrm{q}, 4 \mathrm{H}, 2 \mathrm{x} \mathrm{OCH}, \mathrm{J}=7 \mathrm{~Hz}), 4.27(\mathrm{~s}$, $2 \mathrm{H},-\mathrm{N}-\mathrm{CH}_{2}-\mathrm{N}$ - of morpholine ring), $4.72(\mathrm{~s}, 1 \mathrm{H}, \mathrm{P}-\mathrm{C}-\mathrm{H}), 6.03(\mathrm{~s}, 1 \mathrm{H}, \mathrm{NH})$ and 7.24-7.31 (m, 4H, of flourophenyl group). ${ }^{13} \mathrm{C}-\mathrm{NMR}$ (75 MHz, DMSO-d6): ( ${ }_{\mathrm{pPM}}$. 155.6, 33.7, 172.0, 19.3, 57.5, 62.2, 16.3, 70.3, 53.2, 66.4, 143.2, 118.9, 116.3 and 155.7 corresponding to $\mathrm{C}_{1}, \mathrm{C}_{2}, \mathrm{C}_{3}, \mathrm{C}_{4}, \mathrm{C}_{5}, \mathrm{C}_{6} \& \mathrm{C}_{8}, \mathrm{C}_{7} \& \mathrm{C}_{9}, \mathrm{C}_{10}, \mathrm{C}_{11} \& \mathrm{C}_{14}, \mathrm{C}_{12} \& \mathrm{C}_{13}, \mathrm{C}_{15}, \mathrm{C}_{16}$ \& $\mathrm{C}_{20}, \mathrm{C}_{17}$ \& $\mathrm{C}_{19}$ and $\mathrm{C}_{18}$ respectively. ${ }^{31} \mathrm{P}-\mathrm{NMR}(161.89 \mathrm{MHz}, \mathrm{DMSO}-\mathrm{d} 6)$ : $\left({ }_{\mathrm{ppM}} .21 .11 \mathrm{~m} / \mathrm{z}=456.19\right.$ for $\mathrm{C}_{20} \mathrm{H}_{30} \mathrm{FN}_{4} \mathrm{O}_{5} \mathrm{P}$. Anal. Found (Calcd) C: 51.83 (52.63), H: 6.12 (6.62), N: 11.67 (12.27), F: 3.56 (4.16), P: 6.09 (6.79).

Diethyl (((4 - chorophenyl) amino) (3 - methyl - 1 - (morpholinomethyl) -5-oxo-4, 5 - dihydro-1H-pyrazol-4-yl) methyl) phosphonates (6b) according to general procedure $\mathrm{C}$ to afford the target compound as a yellow solid (0.964gr) with the following characteristic: Yield $68 \%$, m p $157-159^{\circ} \mathrm{C}$. IR $\left(\mathrm{KBr} \mathrm{cm}^{-1}\right)$ 3420-3386 (N-H), 3055 (stretching of Ar$\mathrm{H}), 2940$ and $2895\left(\mathrm{CH}_{3} \& \mathrm{CH}_{2}\right.$ of aliphatic-CH), $1675(\mathrm{C}=\mathrm{O}), 1478-1375$ (stretching vibrations of pyrazolone ring), $1254(\mathrm{P}=\mathrm{O}), 1145(\mathrm{C}-\mathrm{O}), 740(\mathrm{P}-\mathrm{O})$ and $730 \mathrm{~cm}^{-1}$ (C-Cl). ${ }^{1} \mathrm{H}-\mathrm{NMR}(400 \mathrm{MHz}, \mathrm{DMSO}-\mathrm{d} 6)$ : ( ${ }_{\text {ppM }} 1.32$ (t, 6H, 2x P-O$\mathrm{CH}_{2}-\mathrm{CH}_{3} \mathrm{~J}=7 \mathrm{~Hz}$ ), $1.94\left(\mathrm{~s}, 3 \mathrm{H}\right.$, of $\mathrm{CH}_{3}$ group), 2.40 (d, $1 \mathrm{H}, \mathrm{CH}$ of pyrazolone ring), $2.50\left(\mathrm{t}, 4 \mathrm{H},-\mathrm{CH}_{2}-\mathrm{N}-\mathrm{CH}_{2}-\mathrm{of}\right.$ morpholine ring $\mathrm{J}=7 \mathrm{~Hz}), 3.65\left(\mathrm{t}, 4 \mathrm{H},-\mathrm{CH}_{2}-\mathrm{O}-\mathrm{CH}_{2}-\right.$ of morpholine $\left.\operatorname{ring} \mathrm{J}=7 \mathrm{~Hz}\right), 4.10(\mathrm{q}, 4 \mathrm{H}, 2 \mathrm{x} \mathrm{OCH}, \mathrm{J}=7 \mathrm{~Hz}), 4.27(\mathrm{~s}$, $2 \mathrm{H},-\mathrm{N}-\mathrm{CH}_{2}-\mathrm{N}$ - of morpholine ring), $4.65(\mathrm{~s}, 1 \mathrm{H}, \mathrm{P}-\mathrm{C}-\mathrm{H}), 5.95(\mathrm{~s}, 1 \mathrm{H}, \mathrm{NH})$ and 7.02-7.25 (m, 4H, of chlorophenyl group). ${ }^{13} \mathrm{C}-\mathrm{NMR}$ (75 MHz, DMSO-d6): ( ${ }_{\mathrm{PPM}}$. 155.6, 33.7, 172.0, 19.3, 57.5, 62.2, 16.3, 70.3, 53.2, 66.4, 145.7, 114.9, 129.6 and 126.1 corresponding to $\mathrm{C}_{1}, \mathrm{C}_{2}, \mathrm{C}_{3}, \mathrm{C}_{4}, \mathrm{C}_{5}, \mathrm{C}_{6} \& \mathrm{C}_{8}, \mathrm{C}_{7} \& \mathrm{C}_{9}, \mathrm{C}_{10}, \mathrm{C}_{11} \& \mathrm{C}_{14}, \mathrm{C}_{12} \& \mathrm{C}_{13}, \mathrm{C}_{15}, \mathrm{C}_{16} \& \mathrm{C}_{20}, \mathrm{C}_{17}$ \& $\mathrm{C}_{19}$ and $\mathrm{C}_{18}$.respectively. ${ }^{31} \mathrm{P}-\mathrm{NMR}(161.89 \mathrm{MHz}, \mathrm{DMSO}-\mathrm{d} 6)$ : $\left(\right.$ ррм. 20.9. $\mathrm{m} / \mathrm{z}=472.16$ for $\mathrm{C}_{20} \mathrm{H}_{30} \mathrm{ClN}_{4} \mathrm{O}_{5} \mathrm{P}$. Anal. Found (Calcd) C: $50.00(50.80), \mathrm{H}: 5.89$ (6.39), N: 11.25 (11.85), Cl: 6.70 (7.50), P: 5.85 (6.55).

Diethyl (((4 - bromophenyl) amino) (3 - methyl-1- (morpholinomethyl) - 5 - oxo - 4, 5-dihydro-1H-pyrazol-4-yl) methyl) phosphonates $6(\mathbf{c})$ according to general procedure $\mathrm{C}$ to afford the target compound as a yellow solid (1.03gr) with the following characteristic: Yield $67 \%$, m p 142-144 ${ }^{\circ} \mathrm{C}$. IR $\left(\mathrm{KBr} \mathrm{cm}^{-1}\right)$ 3420-3390 (N-H), 3065 (stretching of Ar$\mathrm{H}), 2940$ and $2895\left(\mathrm{CH}_{3} \& \mathrm{CH}_{2}\right.$ of aliphatic- $\left.\mathrm{CH}\right), 1680(\mathrm{C}=\mathrm{O}), 1478-1375$ (stretching vibrations of pyrazolone ring), $1259(\mathrm{P}=\mathrm{O}), 1148(\mathrm{C}-\mathrm{O}), 752(\mathrm{P}-\mathrm{O})$ and $650 \mathrm{~cm}^{-1}$ (C-Br). ${ }^{1} \mathrm{H}-\mathrm{NMR}(400 \mathrm{MHz}, \mathrm{DMSO}-\mathrm{d} 6)$ : ( ppM $^{1.32}$ (t, 6H, 2x P-O$\mathrm{CH}_{2}-\mathrm{CH}_{3} \mathrm{~J}=7 \mathrm{~Hz}$ ), 1.94 (s, 3H, of $\mathrm{CH}_{3}$ group), 2.40 (d, 1H, CH of pyrazolone ring), 2.50 (t, $4 \mathrm{H},-\mathrm{CH}_{2}-\mathrm{N}-\mathrm{CH}_{2}-\mathrm{of}$ morpholine ring $\mathrm{J}=7 \mathrm{~Hz}), 3.65\left(\mathrm{t}, 4 \mathrm{H},-\mathrm{CH}_{2}-\mathrm{O}-\mathrm{CH}_{2}\right.$-of morpholine ring $\left.\mathrm{J}=7 \mathrm{~Hz}\right), 4.10(\mathrm{q}, 4 \mathrm{H}, 2 \mathrm{x} \mathrm{OCH}, \mathrm{J}=7 \mathrm{~Hz}), 4.27(\mathrm{~s}$, 
$2 \mathrm{H},-\mathrm{N}-\mathrm{CH}_{2}-\mathrm{N}$ - of morpholine ring), $4.60(\mathrm{~s}, 1 \mathrm{H}, \mathrm{P}-\mathrm{C}-\mathrm{H}), 5.90(\mathrm{~s}, 1 \mathrm{H}, \mathrm{NH})$ and 7.0-7.20 (m, 4H, of bromophenyl group). ${ }^{13} \mathrm{C}-\mathrm{NMR}$ (75 MHz, DMSO-d6): ( 132.4 and 115.1 corresponding to $\mathrm{C}_{1}, \mathrm{C}_{2}, \mathrm{C}_{3}, \mathrm{C}_{4}, \mathrm{C}_{5}, \mathrm{C}_{6} \& \mathrm{C}_{8}, \mathrm{C}_{7} \& \mathrm{C}_{9}, \mathrm{C}_{10}, \mathrm{C}_{11} \& \mathrm{C}_{14}, \mathrm{C}_{12} \& \mathrm{C}_{13}, \mathrm{C}_{15}, \mathrm{C}_{16}$ \& $\mathrm{C}_{20}, \mathrm{C}_{17}$ \& $\mathrm{C}_{19}$ and $\mathrm{C}_{18}$.respectively. ${ }^{31} \mathrm{P}-\mathrm{NMR}(161.89 \mathrm{MHz}, \mathrm{DMSO}-\mathrm{d6})$ : $\left({ }_{\mathrm{PpM}} .19 .90 \mathrm{~m} / \mathrm{z}=516.11\right.$ for $\mathrm{C}_{20} \mathrm{H}_{30} \mathrm{BrN}_{4} \mathrm{O}_{5} \mathrm{P}$. Anal. Found (Calcd) C: 45.63 (46.43), H: 5.34 (5.84), N: 10.23 (10.83), Br: 14.84 (15.44), P: 4.79 (5.49).

Diethyl ((3 - methyl - 1 - (morpholinomethyl) - 5 - oxo - 4, 5-dihydro - 1 H - pyrazol - 4 - yl) ((4-(trifluoromethyl) phenyl) amino) methyl) phosphonates $6(d)$ according to general procedure $\mathrm{C}$ to afford the target compound as a yellow solid (0.987gr) with the following characteristic: Yield $65 \%$, m p $183-185^{\circ} \mathrm{C}$. IR $\left(\mathrm{KBr} \mathrm{cm}^{-1}\right) 3422-3392(\mathrm{~N}-\mathrm{H}), 3067$ (stretching of Ar-H), 2940 and $2895\left(\mathrm{CH}_{3} \& \mathrm{CH}_{2}\right.$ of aliphatic- $\left.\mathrm{CH}\right), 1673(\mathrm{C}=\mathrm{O}), 1478-1375$ (stretching vibrations of pyrazolone ring), $1256(\mathrm{P}=\mathrm{O}), 1150(\mathrm{C}-\mathrm{O}), 1110(\mathrm{C}-\mathrm{F})$ and $755 \mathrm{~cm}^{-1}$ (P-O). ${ }^{1} \mathrm{H}-\mathrm{NMR}$ (400MHz, DMSO-d6): ( ${ }_{\text {pPM }} 1.32$ (t, 6H, 2x P-O-CH $\left.-\mathrm{CH}_{3} \mathrm{~J}=7 \mathrm{~Hz}\right), 1.94\left(\mathrm{~s}, 3 \mathrm{H}\right.$, of $\mathrm{CH}_{3}$ group), 2.40 (d, 1H, CH of pyrazolone ring), 2.50 (t, 4H, - $\mathrm{CH}-\mathrm{N}-$ $\mathrm{CH}_{2}$ of morpholine ring $\left.\mathrm{J}=7 \mathrm{~Hz}\right), 3.65\left(\mathrm{t}, 4 \mathrm{H},-\mathrm{CH}_{2}-\mathrm{O}-\mathrm{CH}_{2}\right.$-of morpholine ring $\left.\mathrm{J}=7 \mathrm{~Hz}\right), 4.10(\mathrm{q}, 4 \mathrm{H}, 2 \mathrm{x} \mathrm{OCH}, \mathrm{J}=7 \mathrm{~Hz})$, $4.27\left(\mathrm{~s}, 2 \mathrm{H},-\mathrm{N}-\mathrm{CH}_{2}-\mathrm{N}\right.$ - of morpholine ring), $4.80(\mathrm{~s}, 1 \mathrm{H}, \mathrm{P}-\mathrm{C}-\mathrm{H}), 6.15(\mathrm{~s}, 1 \mathrm{H}, \mathrm{NH})$ and $7.25-7.60(\mathrm{~m}, 4 \mathrm{H}$, of triflouromethylphenyl group). ${ }^{13} \mathrm{C}-\mathrm{NMR}$ (75 MHz, DMSO-d6): (ррм. 155.6, 33.7, 172.0, 19.3, 57.5, 62.2, 16.3, 70.3, 53.2, 66.4, 150.9, 113.8, 125.9, 124.9 and 124.1 corresponding to $\mathrm{C}_{1}, \mathrm{C}_{2}, \mathrm{C}_{3}, \mathrm{C}_{4}, \mathrm{C}_{5}, \mathrm{C}_{6} \& \mathrm{C}_{8}, \mathrm{C}_{7} \& \mathrm{C}_{9}, \mathrm{C}_{10}, \mathrm{C}_{11} \& \mathrm{C}_{14}, \mathrm{C}_{12}$ $\& \mathrm{C}_{13}, \mathrm{C}_{15}, \mathrm{C}_{16} \& \mathrm{C}_{20}, \mathrm{C}_{17} \& \mathrm{C}_{19}, \mathrm{C}_{18}$ and $\mathrm{C}_{21}$ respectively. ${ }^{31} \mathrm{P}-\mathrm{NMR}$ (161.89 MHz, DMSO-d6): ( ${ }_{\mathrm{ppM}}$. 26.5. $\mathrm{m} / \mathrm{z}=506.19$ for $\mathrm{C}_{21} \mathrm{H}_{30} \mathrm{~F}_{3} \mathrm{~N}_{4} \mathrm{O}_{5} \mathrm{P}$. Anal. Found (Calcd) C: 49.00 (49.80), H: 5.47 (5.97), N: 10.46 (11.06), F: 10.65 (11.25), P: 5.42 (6.12).

Diethyl (((4-fluorophenyl) amino) (3-methyl-1- (4-methyl piperazin-1-yl) methyl) - 5 - oxo - 4, 5 - dihydro - $1 \mathrm{H}$ pyrazol - 4 - yl) methyl) phosphonates 6(e) according to general procedure $\mathrm{C}$ to afford the target compound as a yellow solid (0.985gr) with the following characteristic: Yield 70\%, m p 149-151 ${ }^{\circ} \mathrm{C}$. IR $\left(\mathrm{KBr} \mathrm{cm}^{-1}\right) 3418-3384(\mathrm{~N}-\mathrm{H}), 3052$ (stretching of Ar-H), 2940 and $2895\left(\mathrm{CH}_{3} \& \mathrm{CH}_{2}\right.$ of aliphatic- $\left.\mathrm{CH}\right), 1674(\mathrm{C}=\mathrm{O}), 1478-1375$ (stretching vibrations of pyrazolone ring), $1254(\mathrm{P}=\mathrm{O}), 1149(\mathrm{C}-\mathrm{O}), 1108(\mathrm{C}-\mathrm{F})$ and $756 \mathrm{~cm}^{-1}$ (P-O). ${ }^{1} \mathrm{H}-\mathrm{NMR}$ (400MHz, DMSO-d6): ( (t, 6H, 2x P-O- $\left.\mathrm{CH}_{2}-\mathrm{CH}_{3} \mathrm{~J}=7 \mathrm{~Hz}\right), 1.94\left(\mathrm{~s}, 3 \mathrm{H}\right.$, of $\mathrm{CH}_{3}$ group), $2.26\left(\mathrm{~s}, 3 \mathrm{H}, \mathrm{CH}_{3}\right.$ attached to piperazin ring), $2.40(\mathrm{~d}, 1 \mathrm{H}$, $\mathrm{CH}$ of pyrazolone ring), $2.65\left(\mathrm{~m}, 8 \mathrm{H},\left(\mathrm{CH}_{2}\right)_{4}\right.$ of piperazine ring), $4.10\left(\mathrm{q}, 4 \mathrm{H}, 2 \mathrm{x} \mathrm{OCH}_{2}, \mathrm{~J}=7 \mathrm{~Hz}\right), 4.27(\mathrm{~s}, 2 \mathrm{H},-\mathrm{N}-\mathrm{CH}-\mathrm{N}-$ of morpholine ring), 4.72 (s, 1H, P-C-H), 6.03 (s, 1H, NH) and 7.24-7.31 (m, 4H, of flourophenyl group). ${ }^{13} \mathrm{C}-\mathrm{NMR}(75$ MHz, DMSO-d6): (ррм. 155.6, 33.7, 172.0, 19.3, 57.5, 62.2, 16.3, 70.0, 52.2, 57.3, 46.6, 143.2, 118.9 , 116.3 and 155.7 corresponding to $\mathrm{C}_{1}, \mathrm{C}_{2}, \mathrm{C}_{3}, \mathrm{C}_{4}, \mathrm{C}_{5}, \mathrm{C}_{6} \& \mathrm{C}_{8}, \mathrm{C}_{7} \& \mathrm{C}_{9}, \mathrm{C}_{10}, \mathrm{C}_{11} \& \mathrm{C}_{14}, \mathrm{C}_{12} \& \mathrm{C}_{13}, \mathrm{C}_{15}, \mathrm{C}_{16}, \mathrm{C}_{17}$ \& $\mathrm{C}_{21}, \mathrm{C}_{18}$ \& $\mathrm{C}_{20}$ and $\mathrm{C}_{19}$.respectively. ${ }^{31} \mathrm{P}-\mathrm{NMR}$ (161.89 MHz, DMSO-d6): $\left({ }_{\mathrm{ppm}}\right.$. 20.10. m/z $=469.2$ for $\mathrm{C}_{21} \mathrm{H}_{33} \mathrm{FN}_{5} \mathrm{O}_{4} \mathrm{P}$. Anal. Found (Calcd) C: 52.92 (53.72), H: 6.58 (7.08), N: 14.32 (14.92), F: 3.45 (4.05), P: 6.00 (6.60).

Diethyl (((4 - chlorophenyl) amino) (3 - methyl - 1 - (4-methyl piperazin-1-yl) methyl) - 5 - oxo - 4, 5-dihydro-1Hpyrazol-4-yl) methyl) phosphonates $6(f)$ according to general procedure $\mathrm{C}$ to afford the target compound as a yellow solid (1.005gr) with the following characteristic: Yield $69 \%$, m p 137-139 ${ }^{\circ} \mathrm{C}$. IR $\left(\mathrm{KBr} \mathrm{cm}^{-1}\right) 3420-3386(\mathrm{~N}-\mathrm{H}), 3055$ (stretching of Ar-H), 2940 and $2895\left(\mathrm{CH}_{3} \& \mathrm{CH}_{2}\right.$ of aliphatic- $\left.\mathrm{CH}\right), 1676(\mathrm{C}=\mathrm{O}), 1478-1375$ (stretching vibrations of pyrazolone ring), $1256(\mathrm{P}=\mathrm{O}), 1150(\mathrm{C}-\mathrm{O}), 747(\mathrm{P}-\mathrm{O})$ and $735 \mathrm{~cm}^{-1}$ (C-Cl). ${ }^{1} \mathrm{H}-\mathrm{NMR}(400 \mathrm{MHz}, \mathrm{DMSO}-\mathrm{d} 6)$ : ( (t, 6H, 2x P-O- $\left.\mathrm{CH}_{2}-\mathrm{CH}_{3} \mathrm{~J}=7 \mathrm{~Hz}\right), 1.94\left(\mathrm{~s}, 3 \mathrm{H}\right.$, of $\mathrm{CH}_{3}$ group), $2.26\left(\mathrm{~s}, 3 \mathrm{H}, \mathrm{CH}_{3}\right.$ attached to piperazin ring), $2.40(\mathrm{~d}, 1 \mathrm{H}$, $\mathrm{CH}$ of pyrazolone ring), $2.65\left(\mathrm{~m}, 8 \mathrm{H},\left(\mathrm{CH}_{2}\right)_{4}\right.$ of piperazine ring), $4.10(\mathrm{q}, 4 \mathrm{H}, 2 \mathrm{x} \mathrm{OCH}, \mathrm{J}=7 \mathrm{~Hz}), 4.27(\mathrm{~s}, 2 \mathrm{H},-\mathrm{N}-\mathrm{CH}-\mathrm{N}-$ of morpholine ring), $4.65(\mathrm{~s}, 1 \mathrm{H}, \mathrm{NH}), 5.95(\mathrm{~s}, 1 \mathrm{H}, \mathrm{P}-\mathrm{C}-\mathrm{H})$ and $7.02-7.20\left(\mathrm{~m}, 4 \mathrm{H}\right.$, of chlorophenyl group). ${ }^{13} \mathrm{C}-\mathrm{NMR}(75$ MHz, DMSO-d6): (рем. 155.6, 33.7, 172.0, 19.3, 57.5, 62.2, 16.3, 70.0, 52.2, 57.3, 46.6, 145.7, 114.9 , 129.6 and 126.1 corresponding to $\mathrm{C}_{1}, \mathrm{C}_{2}, \mathrm{C}_{3}, \mathrm{C}_{4}, \mathrm{C}_{5}, \mathrm{C}_{6} \& \mathrm{C}_{8}, \mathrm{C}_{7} \& \mathrm{C}_{9}, \mathrm{C}_{10}, \mathrm{C}_{11} \& \mathrm{C}_{14}, \mathrm{C}_{12} \& \mathrm{C}_{13}, \mathrm{C}_{15}, \mathrm{C}_{16}, \mathrm{C}_{17}$ \& $\mathrm{C}_{21}, \mathrm{C}_{18}$ \& $\mathrm{C}_{20}$ and $\mathrm{C}_{19}$..respectively. ${ }^{31} \mathrm{P}-\mathrm{NMR}\left(161.89 \mathrm{MHz}\right.$, DMSO-d6): $\left(\right.$ ррм. $19.80 . \mathrm{m} / \mathrm{z}=485.20$ for $\mathrm{C}_{21} \mathrm{H}_{33} \mathrm{ClN}_{5} \mathrm{O}_{4} \mathrm{P}$. Anal. Found (Calcd) C: 51.10(51.90), H: 6.34(6.84), N: 13.81 (14.41), Cl: 6.50 (7.30), P: 5.67 (6.37). 

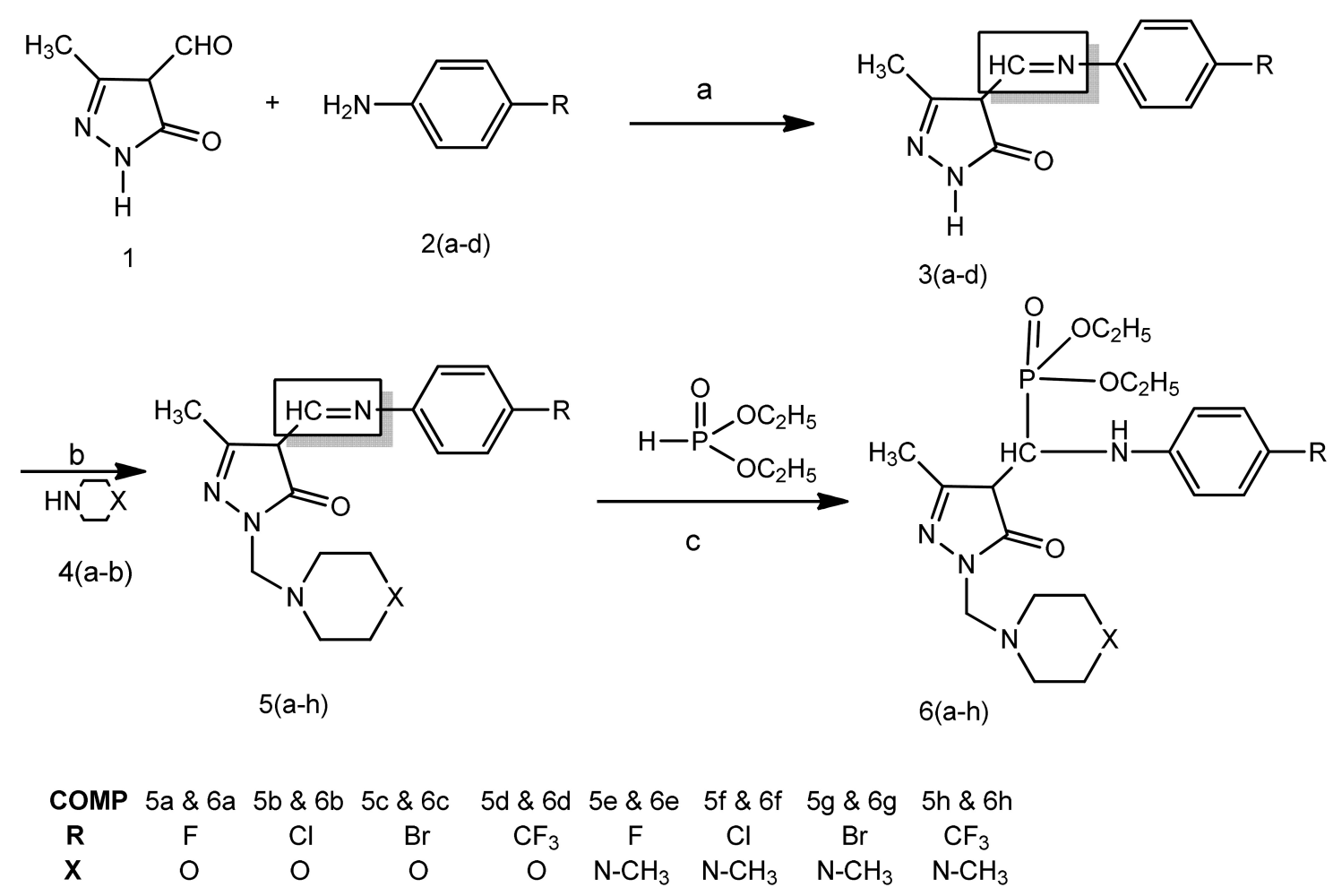

\section{Reagents and conditions:}

(a) Addition of alcohol, acetic acid and heated on a steam bath for

5-6 hours at $100^{\circ} \mathrm{C}$, after standing for 24 hours at R.T.

(b) Addition of $\mathrm{HCHO}$, DMF in ice cold condition and stirred for 2 hours, left over night at R.T.

(c) Anhydrous toluene, stirred at R.T.for 0.5hours, the reaction heated under refluxed for 4-6 hours.

Scheme-1. Synthesis of Mannich bases of Pyrazole-5-one phosphonates 6(a-h)

Diethyl (((4-bromophenyl) amino) (3 - methyl - 1 - (4-methyl piperazin-1-yl) methyl) - 5 - oxo - 4, 5 - dihydro-1Hpyrazol-4-yl) methyl) phosphonates $6(\mathrm{~g})$ according to general procedure $\mathrm{C}$ to afford the target compound as a yellow solid (1.007gr) with the following characteristic: Yield $65 \%, \mathrm{~m} \mathrm{p} 129-131^{\circ} \mathrm{C}$. IR $\left(\mathrm{KBr} \mathrm{cm}^{-1}\right) 3422-3392(\mathrm{~N}-\mathrm{H}), 3065$ (stretching of Ar-H), 2940 and $2895\left(\mathrm{CH}_{3} \& \mathrm{CH}_{2}\right.$ of aliphatic-CH), $1678(\mathrm{C}=\mathrm{O}), 1478-1375$ (stretching vibrations of pyrazolone ring), 1254 ( $\mathrm{P}=\mathrm{O}), 1152$ (C-O), $743(\mathrm{P}-\mathrm{O})$ and $655 \mathrm{~cm}^{-1}$ (C-Br). ${ }^{1} \mathrm{H}-\mathrm{NMR}$ (400MHz, DMSO-d6): ( $\left(\mathrm{t}, 6 \mathrm{H}, 2 \mathrm{x} \mathrm{P}-\mathrm{O}-\mathrm{CH}_{2}-\mathrm{CH}_{3} \mathrm{~J}=7 \mathrm{~Hz}\right), 1.94\left(\mathrm{~s}, 3 \mathrm{H}\right.$, of $\mathrm{CH}_{3}$ group), $2.26\left(\mathrm{~s}, 3 \mathrm{H}, \mathrm{CH}_{3}\right.$ attached to piperazin ring), $2.40(\mathrm{~d}, 1 \mathrm{H}$, $\mathrm{CH}$ of pyrazolone ring), $2.65\left(\mathrm{~m}, 8 \mathrm{H},\left(\mathrm{CH}_{2}\right)_{4}\right.$ of piperazine ring), $4.10\left(\mathrm{q}, 4 \mathrm{H}, 2 \mathrm{x} \mathrm{OCH}_{2}, \mathrm{~J}=7 \mathrm{~Hz}\right), 4.27\left(\mathrm{~s}, 2 \mathrm{H},-\mathrm{N}-\mathrm{CH}_{2}-\mathrm{N}-\right.$ of morpholine ring), $4.65(\mathrm{~s}, 1 \mathrm{H}, \mathrm{NH}), 5.95(\mathrm{~s}, 1 \mathrm{H}, \mathrm{P}-\mathrm{C}-\mathrm{H})$ and 7.0-7.20 (m, 4H, of bromophenyl group). ${ }^{13} \mathrm{C}-\mathrm{NMR}(75$ MHz, DMSO-d6): (ррм. 155.6, 33.7, 172.0, 19.3, 57.5, 62.2, 16.3, 70.0, 52.2, 57.3, 46.6, 146.6, 114.5, 132.4 and 115.1 corresponding to $\mathrm{C}_{1}, \mathrm{C}_{2}, \mathrm{C}_{3}, \mathrm{C}_{4}, \mathrm{C}_{5}, \mathrm{C}_{6} \& \mathrm{C}_{8}, \mathrm{C}_{7} \& \mathrm{C}_{9}, \mathrm{C}_{10}, \mathrm{C}_{11} \& \mathrm{C}_{14}, \mathrm{C}_{12} \& \mathrm{C}_{13}, \mathrm{C}_{15}, \mathrm{C}_{16}, \mathrm{C}_{17} \& \mathrm{C}_{21}, \mathrm{C}_{18}$ \& $\mathrm{C}_{20}$ and $\mathrm{C}_{19}$ respectively. ${ }^{31} \mathrm{P}-\mathrm{NMR}\left(161.89 \mathrm{MHz}\right.$, DMSO-d6): ${ }_{\mathrm{pPM}} \cdot 19.60 . \mathrm{m} / \mathrm{z}=529.15$ for $\mathrm{C}_{21} \mathrm{H}_{33} \mathrm{BrN}_{5} \mathrm{O}_{4} \mathrm{P}$. Anal. Found (Calcd) $\mathrm{C}$ : 46.75 (47.55), H: 5.77 (6.27), N: 13.60 (13.20), Br: 14.40 (15.00), P: 5.14 (5.84).

Diethyl ((3-methyl - 1 - (4 -methyl piperazin - 1 - yl) -methyl - 5 - oxo - 4, 5 -dihydro-1H-pyrazol - 4 - yl) ((4 (trifluoromethyl) phenyl) amino) methyl) phosphonates $6(\boldsymbol{h})$ according to general procedure $\mathrm{C}$ to afford the target compound as a yellow solid (1.168gr) with the following characteristic: Yield $75 \%, \mathrm{~m} \mathrm{p} 167-169^{\circ} \mathrm{C} . \mathrm{IR}\left(\mathrm{KBr} \mathrm{cm}^{-1}\right)$ 3422-3392 (N-H), 3067 (stretching of Ar-H), 2940 and $2895\left(\mathrm{CH}_{3} \& \mathrm{CH}_{2}\right.$ of aliphatic-CH), $1680(\mathrm{C}=\mathrm{O}), 1478-1375$ 
(stretching vibrations of pyrazolone ring), $1254(\mathrm{P}=\mathrm{O}), 1115(\mathrm{C}-\mathrm{O}), 1108(\mathrm{C}-\mathrm{F})$ and $756 \mathrm{~cm}^{-1}(\mathrm{P}-\mathrm{O}) .{ }^{1} \mathrm{H}-\mathrm{NMR}$ (400MHz, DMSO-d6): ( ${ }_{\text {PPM }} 1.32\left(\mathrm{t}, 6 \mathrm{H}, 2 \mathrm{x} \mathrm{P}-\mathrm{O}-\mathrm{CH}_{2}-\mathrm{CH}_{3} \mathrm{~J}=7 \mathrm{~Hz}\right), 1.94$ (s, 3H, of $\mathrm{CH}_{3}$ group), $2.26\left(\mathrm{~s}, 3 \mathrm{H}, \mathrm{CH}_{3}\right.$ attached to piperazin ring), $2.40\left(\mathrm{~d}, 1 \mathrm{H}, \mathrm{CH}\right.$ of pyrazolone ring), $2.65\left(\mathrm{~m}, 8 \mathrm{H},\left(\mathrm{CH}_{2}\right)_{4}\right.$ of piperazine $\left.\operatorname{ring}\right), 4.10(\mathrm{q}, 4 \mathrm{H}, 2 \mathrm{x} \mathrm{OCH}$, $\mathrm{J}=7 \mathrm{~Hz}), 4.27\left(\mathrm{~s}, 2 \mathrm{H},-\mathrm{N}-\mathrm{CH}_{2}-\mathrm{N}\right.$ - of morpholine ring), 4.65 (s, 1H, NH), 5.95 (s, 1H, P-C-H) and 7.25-7.60 (m, 4H, of triflouromethyl phenyl group). ${ }^{13} \mathrm{C}-\mathrm{NMR}$ (75 MHz, DMSO-d6): (ерм. 155.6, 33.7, 172.0, 19.3, 57.5, 62.2, 16.3, 70.0, 52.2, 57.3, 46.6, 150.9, 113.8, 125.9, 124.9 and 124.1 corresponding to $\mathrm{C}_{1}, \mathrm{C}_{2}, \mathrm{C}_{3}, \mathrm{C}_{4}, \mathrm{C}_{5}, \mathrm{C}_{6} \& \mathrm{C}_{8}, \mathrm{C}_{7} \& \mathrm{C}_{9}, \mathrm{C}_{10}, \mathrm{C}_{11} \&$ $\mathrm{C}_{14}, \mathrm{C}_{12} \& \mathrm{C}_{13}, \mathrm{C}_{15}, \mathrm{C}_{16}, \mathrm{C}_{17} \& \mathrm{C}_{21}, \mathrm{C}_{18} \& \mathrm{C}_{20}, \mathrm{C}_{19}$ and $\mathrm{C}_{22}$.respectively. ${ }^{31} \mathrm{P}-\mathrm{NMR}(161.89 \mathrm{MHz}, \mathrm{DMSO}-\mathrm{d} 6):(\mathrm{PPM} \cdot 24.5 . \mathrm{m} / \mathrm{z}$ $=519.22$ for $\mathrm{C}_{22} \mathrm{H}_{33} \mathrm{~F}_{3} \mathrm{~N}_{5} \mathrm{O}_{4} \mathrm{P}$. Anal. Found (Calcd) $\mathrm{C}: 50.06$ (50.86), H: 5.90 (6.40), N: 12.78 (13.48), F: 10.37 (10.97), P: 5.26 (5.96).

\section{Biological Activity}

The antimicrobial activity [13] of these newly synthesized compounds was performed according to disc diffusion method, as recommended by the National Committee for Clinical Laboratory. The synthesized compounds were used at the concentration of $250 \mu \mathrm{g} / \mathrm{ml} \mathrm{DMF}$ as a solvent [14].

\section{Anti-Bacterial Activity}

The antibacterial activity of Mannich bases containing Pyrazole-5-one Phosphonates 6(a-h) were screened against the Staphylococcus aureus and Bacillus cerus (gram positive) and Escherichia coli, Pseudomonasaeruginosa (gram negative) organisms. Most of the compounds exhibit good antibacterial activity against both bacteria. The presence of fluoro (6d, 6h) and chloro (6b, 6f) were showed more activity than other substituted compounds. Here Amoxicillin is tested as reference compound to compare the activity. The anti-bacterial activity was shown in the Table 1.1

Table 1.1. Antifungal activity (Diameter zone of Inhibition in mm) Compounds of 6(a-h).

\begin{tabular}{|c|c|c|c|c|}
\hline \multirow{2}{*}{ COMP NO } & \multicolumn{4}{|c|}{ Zone of inhibition(mm) (250 $\boldsymbol{\mu g}$ / disc) } \\
\cline { 2 - 5 } & $\begin{array}{c}\text { Staphylococcus aureus } \\
\text { NCCS2 079 }\end{array}$ & $\begin{array}{c}\text { Bacillus cereus } \\
\text { NCCS 2106 }\end{array}$ & $\begin{array}{c}\text { Escherichia coli } \\
\text { NCCS 2065 }\end{array}$ & $\begin{array}{c}\text { Pseudomonas aeruginosa } \\
\text { NCCS 2200 }\end{array}$ \\
\hline 6a & 12 & 14 & 11 & 13 \\
\hline 6b & 13 & 15 & 12 & 14 \\
\hline $6 \mathrm{c}$ & 10 & 12 & 09 & 17 \\
\hline 6d & 16 & 18 & 15 & 11 \\
\hline $6 \mathrm{e}$ & 10 & 12 & 09 & 13 \\
\hline 6f & 12 & 14 & 11 & 09 \\
\hline 6g & 08 & 10 & 07 & 15 \\
\hline 6h & 14 & 16 & 13 & 22 \\
\hline Amoxicillin & 21 & 27 & 24 & \\
\hline
\end{tabular}

\section{Anti-Fungal Activity}

The anti-fungal activity of Mannich bases containing Pyrazole-5-one Phosphonates 6(a-h) was screened against the Aspergillus niger and Candida albicans. Most of the compounds exhibit good antifungal activity against both fungai. The presence of fluoro $(\mathbf{6 d}, \mathbf{6 h})$ and chloro $(\mathbf{6 b}, \mathbf{6 f})$ were showed more activity than other substituted compounds. Here Ketoconazole is tested as reference compound to compare the activity. The anti-fungal activity was shown in the Table 1.2 .

Table 1.2. Antifungal activity (Diameter zone of Inhibition in mm) Compounds of 6(a-h).

\begin{tabular}{|c|c|c|}
\hline \multirow{2}{*}{ COMP NO } & \multicolumn{2}{|c|}{ Zone of inhibition(mm) (250 $\boldsymbol{\mu g} /$ disc) } \\
\cline { 2 - 3 } & $\begin{array}{c}\text { Aspergillus niger } \\
\text { NCCS 1196 }\end{array}$ & $\begin{array}{c}\text { Candida albicans } \\
\text { NCCS 3471 }\end{array}$ \\
\hline 6a & 11 & 13 \\
\hline 6b & 14 & 16 \\
\hline 6c & 10 & 12 \\
\hline 6d & 17 & 19 \\
\hline 6e & 09 & 11 \\
\hline 6f & 12 & 14 \\
\hline
\end{tabular}


(Table $\square$ प) contd....

\begin{tabular}{|c|c|c|}
\hline \multirow{2}{*}{ COMP NO } & \multicolumn{2}{|c|}{ Zone of inhibition(mm) (250 $\boldsymbol{\mu g} /$ disc) } \\
\cline { 2 - 3 } & $\begin{array}{c}\text { Aspergillus niger } \\
\text { NCCS 1196 }\end{array}$ & $\begin{array}{c}\text { Candida albicans } \\
\text { NCCS 3471 }\end{array}$ \\
\hline $6 \mathrm{~g}$ & 08 & 10 \\
\hline $6 \mathrm{~h}$ & 14 & 16 \\
\hline Ketoconazole & 22 & 25 \\
\hline
\end{tabular}

\section{CONFLICT OF INTEREST}

The authors confirm that this article content has no conflict of interest.

\section{ACKNOWLEDGEMENTS}

The author V. Esther Rani thanks to U G C - S A P and U G C - B S R, New Delhi for financial assistance. They are also thankful to IICT Hyderabad and CDRI Lucknow for spectral and analytical data.

\section{SUPPLEMENTARY MATERIAL}

Supplementary material is available on the publishers Web site along with the published article.

\section{REFERENCES}

[1] Osman SA. Mohamed, Pyrazolone as a core unit biological evaluation agent minireview. Egypt J Chem 1987; $30: 481$.

[2] Jolly VS. Pathak, Manish. Brief review on Chemistry of Pyrazoline and its derivatives. J Indian Chem Soc 1991; $68: 304$.

[3] Ankhiwala MD, Hathi MV. Novel Synthesis of 1, 3, 5-trisubstituted 2-pyrazolines. Indian J Heterocycl Chem 1996; 5: 229.

[4] Subbanwad R, Vibhute YB. Synthesis antimicrobial and antifungal activity of novel pyrazolines derivatives. J Indian Chem Soc 1992; 69: 337.

[5] Breuer E, Heartly FR, Eds. The Chemistry of Organophosphorus compounds. Newyork: John Wiley \& Sons. 1996.

[6] Faraci WS, Yang BV, O’Rourke D, Spencer RW. Inhibition of Helicobacter pylori urease by phenyl phosphorodiamidates: mechanism of action. Bioorg Med Chem 1995; 3(5): 605-10.

[http://dx.doi.org/10.1016/0968-0896(95)00043-G] [PMID: 7648208]

[7] Fest C, Schmidt KJ. The Chemistry of Organophosphorus Pesticides. Heidelberg: Springer Berlin 1982. [http://dx.doi.org/10.1007/978-3-642-68441-8]

[8] Nivarkar M, Gupta AK, Kaushik MP. Efficient method for synthesis of diazaphospholidines. Tetrahedron Lett 2004; 45 : 6863.

[9] Haranadha Reddy Y, Siva kumar B, Chandrashekar Reddy G, Dadapear E, Subramanyam Reedy K. Synthesis and bioassay of $\alpha$-aminophosphonates. Der chemical Sinica 2012; 3(4): 817-23.

[10] Mehellou Y, McGuigan C, Brancale A, Balzarini J. Design, synthesis, and anti-HIV activity of 2',3'-didehydro-2',3'-dideoxyuridine (d4U), 2',3'-dideoxyuridine (ddU) phosphoramidate 'ProTide' derivatives. Bioorg Med Chem Lett 2007; 17(13): 3666-9. [http://dx.doi.org/10.1016/j.bmcl.2007.04.043] [PMID: 17485204]

[11] Guigan CM, Thiery JC, Daverio F, Jiang WJ, Davies G, Mason M. Method for Synthesis of diazaphopholidines toxicological evaluation. Bioorg Med Chem 2005; 13: 3219. [PMID: 15809157]

[12] Sikoski JA, Miller MJ, Barccolino DS, et al. In vitro and in vivo Inhibition of Hydroxyapatite formation and dissolution by bisacylphosphonates and bishydroxyiminophosphonates. Phosphorus Sulfur Silicon Relat Elem 1993; 76: 115-8.

[13] Arun K. Wahi, Arti Singh. Synthesis characterization and antibacterial activity of some oxadiazole substituted triazole derivative. Der Chemica Sinica 2011;2(3): 11-9.

[14] Bharadwaj S, Parashar B, Parashar N, Sharma VK. Microwave assisted Synthesis and pharmacological evaluation of some 1, 3, 4-oxadiazole derivatives. Scholar Research Library. Achieves of App Sci Res 2011; 3(2): 558-67.

(C) Rani and Ravindranath; Licensee Bentham Open.

This is an open access article licensed under the terms of the Creative Commons Attribution-Non-Commercial 4.0 International Public License (CC BY-NC 4.0) (https://creativecommons.org/licenses/by-nc/4.0/legalcode), which permits unrestricted, non-commercial use, distribution and reproduction in any medium, provided the work is properly cited. 